\title{
RESEARCH
}

Open Access

\section{Nonadherent culture method promotes MSC-mediated vascularization in myocardial infarction via miR-519d/VEGFA pathway}

Baoping Deng ${ }^{1,2}$, Xianlan Zhang ${ }^{2}$, Yi Liang ${ }^{1}$, Haiming Jiang ${ }^{1}$, Weizhao Huang ${ }^{1}$, Yinmeng Wu $^{1}$ and Weiping Deng ${ }^{3^{*}}$

\begin{abstract}
Background: Mesenchymal stem cells (MSCs) can provide therapeutic benefits for myocardial infarction (MI) recovery; however, the molecular mechanism by which MSCs improve the heart function is unclear.

Methods: Microarray analysis was performed to examine the expression profiling of human MSCs (hMSCs) grown as adherent cultures (AC-hMSCs) or nonadherent cultures on ultra-low-adherent plates (nonAC-hMSCs). Real-time quantitative polymerase chain reaction (RT-qPCR), western blotting, and enzyme-linked immunosorbent assays (ELISA) were used to assess VEGFA expression and secretion in the AC-hMSCs and nonAC-hMSCs. The paracrine effect of VEGFA-overexpressing AC-MSCs (AC-VEGFA-hMSCs) or VEGFA-knockdown nonAC-hMSCs (nonAC-shVEGFAhMSCs) on the angiogenic ability of human umbilical vein endothelial cells (HUVECs) was evaluated using tube formation assay. AC-VEGFA-hMSCs or nonAC-shVEGFA-hMSCs were transplanted into myocardial infarction rats to investigate the therapeutic effect of AC-VEGFA-hMSCs or nonAC-shVEGFA-hMSCs. Luciferase reporter assay was used to confirm the association of VEGFA with miR-519d.

Results: Microarray analysis revealed that VEGFA is downregulated in AC-hMSCs compared to nonAC-hMSCs. Functional assays revealed that high levels of VEGFA produced from AC-VEGFA-hMSCs increased the tube formation capacity of HUVECs in vitro, improved angiogenesis and cardiac performance, and reduced infarct size in a rat MI model. Low levels of VEGFA secretion from nonAC-shVEGFA-hMSCs had the opposite effects. Mechanistically, we found that miR-519d directly targets VEGFA. High levels of VEGFA secreted from VEGFA-overexpressing nonAChMSCs abolished the repressive effect of miR-519d on HUVEC angiogenesis.

Conclusion: Our findings indicate that nonadherent culture-induced secretion of VEGFA plays an important role in MSCs via the miR-519d/NEGFA pathway and may provide a novel therapeutic strategy for Ml treatment.
\end{abstract}

Keywords: Mesenchymal stem cells, Myocardial infarction, VEGFA, Angiogenesis, miR-519d, Nonadherent culture

\footnotetext{
*Correspondence: weipingdong2020@163.com

${ }^{3}$ Department of Gastroenterology, Taihe Hospital, Hubei University of

Medicine, 32 Ren Min South Road, Shiyan 442000, Hubei, People's Republic of China

Full list of author information is available at the end of the article
}

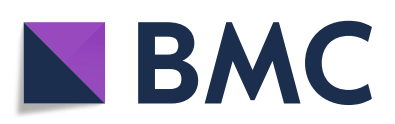

(- The Author(s). 2020 Open Access This article is licensed under a Creative Commons Attribution 4.0 International License, which permits use, sharing, adaptation, distribution and reproduction in any medium or format, as long as you give appropriate credit to the original author(s) and the source, provide a link to the Creative Commons licence, and indicate if changes were made. The images or other third party material in this article are included in the article's Creative Commons licence, unless indicated otherwise in a credit line to the material. If material is not included in the article's Creative Commons licence and your intended use is not permitted by statutory regulation or exceeds the permitted use, you will need to obtain permission directly from the copyright holder. To view a copy of this licence, visit http://creativecommons.org/licenses/by/4.0/ The Creative Commons Public Domain Dedication waiver (http://creativecommons.org/publicdomain/zero/1.0/) applies to the data made available in this article, unless otherwise stated in a credit line to the data. 


\section{Introduction}

Myocardial infarction (MI) is a major cause of mortality and disability in the world [1]. Pharmacologic intervention and conventional revascularization techniques, such as coronary artery bypass grafting, balloon angioplasty, and stenting, can restore blood flow and maintain myocardial viability and function; however, these treatment methods cannot salvage the dying myocardium or repair cardiac function [2]. In recent years, stem cell therapy has become a promising approach to treat MI, as it has the potential to restore damaged myocardium [3, 4]. Mesenchymal stem cells (MSCs) exist in the bone marrow, umbilical cord blood, adipose, and many other tissues [5] and have multilineage differentiation potential, self-renewal capacity, and immunomodulatory properties [6], while posing a low immunorejection risk [7]. Thus, MSCs have been commonly used in experimental research and clinical trials for treating MI. Often for this application, a large amount of MSCs must be isolated from the same tissues and expanded in plastic adherent culturing containers $[7,8]$. It was recently reported that in vitro expansion in conventional monolayer cultures can alter the phenotype of MSCs, which may cause cell trapping within the lung and a low rate of delivery to target organs [9-12]. Qian et al. [13] showed that primary MSCs lacked CD44, whereas culture-expanded MSCs acquired CD44 expression. Compared to primary MSCs, cultured MSCs with high levels of CD44 displayed decreased targeting to the bone marrow [13]. Our previous research revealed that stem cell antigen 1 (Sca-1) is expressed at higher levels in adherent cultured mouse MSCs (AC-mMSCs) compared to mMSCs in nonadherent cultures maintained in ultra-low-adherent plates (nonAC-mMSCs) [14]. Sca- $1^{+}$mMSCs play a crucial role in improving cardiac function in MI [14, 15]. Compared with nonadherent cultured human MSCs (nonAChMSCs), adherent cultured hMSCs (AC-hMSCs) with high protein expression of caspase-3, caspase-7, and caspase-9 had a marked decrease in cell apoptosis [16]. Therefore, it is important to investigate the changes in MSC phenotype that occur during in vitro cultivation.

MSC therapy with angiogenic factors holds a great promise for ischemic disease treatment due to neovascularization [17, 18]. Vascular endothelial growth factor A (VEGFA) is a major driver of angiogenesis and vasculogenesis [19]. VEGFA could regulate angiogenesis and myogenesis in cardiac repair $[20,21]$. A growing body of research demonstrated that VEGFA promotes MSC viability in the infarcted hearts via decreasing cellular stress and enhancing cell survival factors [22, 23]. Therefore, it is anticipated that VEGFA-gene-modified MSCs may provide a potentially valuable approach for MI treatment due to increase survival and angiogenic capacity.
In this study, we identified that VEGFA expression is significantly higher in nonAC-hMSCs than in AChMSCs, as detected by microarray analysis. The increased levels of VEGFA secreted from VEGFAoverexpressing AC-hMSCs (AC-VEGFA-hMSCs) facilitated tube formation of human umbilical vein endothelial cells (HUVECs), while the decreased levels of VEGFA secreted by nonAC-hMSCs with VEGFA knockdown (nonAC-shVEGFA-hMSCs) led to the opposite effect. In a rat MI model, the increased levels of VEGFA released from AC-VEGFA-hMSCs promoted angiogenesis, decreased infarct size, and improved myocardial function, while the decreased levels of VEGFA secreted by nonAC-shVEGFA-hMSCs reduced angiogenesis, increased infarct size, and impaired cardiac remodeling. Further studies on the mechanism underlying these changes indicated that VEGFA is a direct target of miR519d. High levels of VEGFA produced by VEGFAoverexpressing nonAC-hMSCs reversed the inhibitory effect of miR-519d on the tube formation capability of HUVECs.

\section{Materials and methods}

Isolation and expansion of hMSCs and in vitro nonadherent culture conditions

hMSCs were isolated from the bone marrow of adult donors (ages 18-25) who underwent orthopedic surgery as described previously [16]. hMSCs were seeded in adherent culture plates (AC-hMSCs) or ultra-low-adherent tissue culture plates (nonAC-hMSCs; Corning ${ }^{\circ}$, Corning, $\mathrm{NY}$ ) and grown in Dulbecco's modified Eagle's medium (DMEM; GIBCO, Grand Island, NY) for $24 \mathrm{~h}$ or $72 \mathrm{~h}$. All cells were incubated in a humidified incubator with $5 \%$ $\mathrm{CO}_{2}$ at $37{ }^{\circ} \mathrm{C}$. This study was approved by the Ethics Committee of Affiliated Hospital of Guilin Medical University (Guilin, China), and informed consent was obtained from all participants.

\section{Flow cytometry}

Cells were harvested, washed, and resuspended. The antibodies included anti-CD11b (BD Biosciences, Franklin Lakes, NJ, USA), anti-CD14 (BD Biosciences), antiCD34 (BD Biosciences), anti-CD45 (BD Biosciences), anti-CD73 (BD Biosciences), anti-CD90 (BD Biosciences), and anti-CD105 (BD Biosciences) were added to the cells at $4{ }^{\circ} \mathrm{C}$ for $1 \mathrm{~h}$. Then, cells were analyzed on a BD FACSCalibur (BD Biosciences) and data was analyzed by BD FACSComp software (BD Biosciences). An isotype control immunoglobulin (BD Biosciences) was used as control.

\section{mRNA microarray analysis}

AC-hMSCs and nonAC-hMSCs grown for $24 \mathrm{~h}$ and $72 \mathrm{~h}$ were sent to the Shanghai Bohao Biotechnological Co., 
Ltd. (Shanghai, China), for mRNA microarray profiling. Microarray data were analyzed using GeneSping software (Agilent Technologies, Santa Clara, CA). The $\log _{2^{-}}$ fold change was determined by calculating gene expression in AC-hMSCs and nonAC-hMSCs. Differentially expressed genes were identified using a $t$ test and Benjamini-Hochberg correction (corrected $P<0.05$ and fold change $>2$ ).

\section{Plasmid construction, oligonucleotide, and transfection}

The full-length VEGFA cDNA was PCR-amplified and inserted into a pcDNA3.1 plasmid. miR-519d mimics, miR-519d inhibitors, and the appropriate controls were purchased from Ribobio (Guangzhou, China). Transient transfection was performed using Lipofectamine 2000 (Invitrogen, Carlsbad, CA) according to the manufacturer's instructions.

\section{Lentiviruses and infection}

VEGFA, shVEGFA, and the corresponding control lentiviruses also carrying the GFP coding sequence were obtained from GeneChem Co., Ltd. (Shanghai, China). AChMSCs and nonAC-hMSCs were transduced with VEGFA or shVEGFA lentiviruses (AC-VEGFA-hMSCs or nonACshVEGFA-hMSCs), respectively. An inverted fluorescence microscope was used to detect GFP expression.

Real-time quantitative polymerase chain reaction (RT-qPCR) Total RNA was extracted from cultured cells using Trizol reagent (Invitrogen). A microRNA Reverse Transcription Kit (Promega, Madison, WI, USA) or a PrimeScript RT-PCR kit (Takara, Dalian, China) was used to synthesize cDNA. RT-qPCR was carried out using SYBR Premix Ex Taq II (TaKaRa). Relative quantification of gene expression was determined using the $2^{-\triangle \Delta \mathrm{Ct}}$ method [24]. The following primers were used: VEGFA primers: forward 5'-GCAGAATCATCACG AAGTGGTG-3', reverse 5'-TCTCGATTGGATGG CAGTAGCT-3'; $\beta$-actin primers: forward $5^{\prime}$-CTCCAT CCTGGCCTCGCTGT-3', reverse 5'-ACTAAGTCAT AGTCCGCCTAGA-3'; miR-519d RT primers: 5'GTCGTATCCAGTGCAGGGTCCGAGGTATTCGCAC TGGATACGACCACTCT-3', PCR primers: forward 5'GCCAAAGTGCCTCCCTTT-3', reverse 5'-GTGCAG GGTCCGAGGT-3'; U6 primers: forward 5'-CTCGCT TCGGCAGCACA-3', reverse 5' ${ }^{\prime}$-AACGCTTCACGAAT TTGCGT-3'.

\section{Enzyme-linked immunosorbent assays (ELISA)}

Conditioned medium (CM) was obtained from different MSC groups and centrifuged for further experiments. Rat heart tissues were homogenized in $20 \mathrm{~mL}$ of PBS, subjected to two freeze-thaw cycles, and centrifuged for further experiments. The secreted levels of VEGFA in the $\mathrm{CM}$ and rat heart tissues were examined using a VEGFA ELISA Kit (R\&D System, Los Angeles, CA) according to the manufacturer's protocol, and the absorbance at $450 \mathrm{~nm}$ was measured using a microplate reader. Each sample was assayed three times.

\section{Tube formation assay}

HUVECs were obtained from the Jennio Biotech (Guangzhou, China). HUVECs $\left(2 \times 10^{4}\right.$ cells) were suspended in CM obtained from different hMSC groups, added to the 96-well plates coated with Matrigel (BD Biosciences, Franklin Lakes, NJ) at a density of $2 \times 10^{4}$ cells/well, and incubated at $37{ }^{\circ} \mathrm{C}$ for $12 \mathrm{~h}$. Images of tubular structures were captured and analyzed using Image-Pro Plus 6.0 software.

\section{Rat MI model and hMSC transplantation}

Male Sprague-Dawley rats weighing 250-350 g were subjected to MI using left anterior descending coronary artery (LADCA) ligation. After anesthetized with 10\% chloral hydrate $(250 \mathrm{mg} / \mathrm{kg}$ weight), the rats were intubated with polyethylene- 16 tube and connected to the rodent ventilator (Harvard Apparatus, Holliston, MA). The chest was opened between the third and fourth ribs, and the pericardium was split to expose the left ventricle (LV), aorta, and left atrium. The LADCA was ligated using a 7-0 polypropylene suture $2 \mathrm{~mm}$ below the edge of the left atrium. Forty-eight rats were randomly divided into six treatment groups: rats that received ACVEGFA-hMSCs $(n=8)$, nonAC-shVEGFA-hMSCs $(n=$ $8)$, AC-hMSCs $(n=8)$, nonAC-hMSCs $(n=8)$, saline (MI group, $n=8$ ), or saline (a sham operation, $n=8$ ) in the infarct border region immediately after LAD ligation. The chest and skin were then closed. All experiments were approved by the Ethics Committee of the Affiliated Hospital of Guilin Medical University.

\section{Cardiac function assessment}

Four weeks after LADCA ligation, an echocardiography was performed using a Vevo 2100 system (Visualsonics, Toronto, ON, Canada) with a $21-\mathrm{MHz}$ transducer. Rats were anesthetized with $10 \%$ chloral hydrate $(250 \mathrm{mg} / \mathrm{kg}$ weight) for the exam. LV end-diastolic and end-systolic dimensions (LVEDD, LVESD) from the parasternal shortaxis view at the papillary muscle were measured using $\mathrm{M}$ mode tracing [25]. LV ejection fraction (LVEF) was calculated using the following formula: $\operatorname{LVEF}(\%)=\left(\mathrm{LVEDD}^{3}\right.$ $\left.\operatorname{LVESD}^{3}\right) / \mathrm{LVEDD}^{3} \times 100 . \mathrm{LV}$ fractional shortening $(\mathrm{LVFS})$ was calculated as LVFS (\%) =(LVEDD - LVESD)/ LVEDD $\times 100$. Measurements were done in triplicate.

\section{Measurement of LV infarct size}

The heart tissues were fixed in 10\% formalin, embedded in paraffin, and cut into $5 \mu \mathrm{m}$ slices. Sections were then 
stained with Masson's trichrome according to the manufacturer's instructions and examined using an optical microscope (Olympus, Tokyo, Japan), and images were acquired using a Retiga CCD camera. Image-Pro Plus 6.0 software was used to measure the infarct area and total LV area of each image. Infarct size was expressed as a percentage of the total LV area.

\section{Immunohistochemical staining}

Sections were dewaxed with xylene and rehydrated with graded ethanol. Subsequently, sections were pretreated with $0.01 \mathrm{M}$ citrate buffer ( $\mathrm{pH}$ 6.0) in a microwave oven at $95{ }^{\circ} \mathrm{C}$ for $20 \mathrm{~min}$ and incubated with $3 \% \mathrm{H}_{2} \mathrm{O}_{2}$ for $15 \mathrm{~min}$ at room temperature. The sections were blocked with $10 \%$ sheep serum for $30 \mathrm{~min}$ and then incubated with anti-Von Willebrand Factor (vWF, Abcam, Cambridge, MA) primary antibody overnight at $4{ }^{\circ} \mathrm{C}$. After washes with PBS, sections were incubated with secondary antibody for 30 $\mathrm{min}$ at room temperature and stained with diaminobenzidine. After counterstaining with hematoxylin, sections were analyzed using an optical microscope (Olympus) and photographed using a digital camera.

\section{Luciferase reporter assay}

The VEGFA 3' UTR, which contains a putative miR519d binding site, was PCR-amplified and inserted into the psiCHECK-2 luciferase reporter plasmid (VEGFA-3' UTR-WT; Promega). Site-directed mutagenesis (Stratagene, La Jolla, CA) was used to construct the mutant VEGFA 3' UTR (Mut), which was then cloned into the psiCHECK-2 luciferase reporter plasmid (VEGFA-3' UTR-Mut). VEGFA-3' UTR-WT or VEGFA-3' UTRMut vectors were transfected into HEK293T cells. Luciferase activity was analyzed $48 \mathrm{~h}$ after transfection using a dual-luciferase reporter assay kit (Promega). Data are represented as the Renilla/firefly luciferase ratio.

\section{Western blotting}

Tissues and cells were lysed with RIPA lysis buffer (Beyotime Biotechnology, Shanghai, China) containing protease inhibitor. Protein extracts were separated by SDS-PAGE and transferred onto a PVDF membrane (Millipore, Billerica, MA). The membrane was blocked with 5\% non-fat milk and incubated with anti-VEGFA primary antibody (Abcam) at $4{ }^{\circ} \mathrm{C}$ overnight, followed by incubation with HRP-conjugated secondary antibody at room temperature. Protein signals were visualized using an ECL detection kit (Millipore).

\section{Statistical analysis}

The data analysis was performed using SPSS 20.0 software. The differences between two groups were evaluated using student's $t$ tests. Values are expressed as the mean \pm standard deviation. $P$ values $<0.05$ were considered statistically significant.

\section{Results}

Increased VEGFA expression and production are observed in nonAC-hMSCs

We first used flow cytometry to identify MSCs and found that hMSCs were positive for CD73, CD90, and CD105, whereas negative for CD11b, CD14, CD34, and CD45 (Additional file 1: Figure S1). Next, microarray analysis was performed in order to profile mRNAs in AC-hMSCs and nonAC-hMSCs at two different time points (Fig. 1a; Additional file 2: Table S1; Additional file 3: Table S2). Two thousand four hundred ninety-six mRNAs were downregulated (1663 genes at $24 \mathrm{~h}$ and 1820 genes at $72 \mathrm{~h}$ ), and 2400 mRNAs were upregulated (1489 genes at $24 \mathrm{~h}$ and 1766 genes at $72 \mathrm{~h}$ ) in nonAC-hMSCs when compared to those in AC-hMSCs (Fig. 1b). The mRNA expression profiles from nonAChMSCs at two different time points showed some overlap, with 987 downregulated mRNAs and 855 upregulated mRNAs shared between both samples (Fig. 1b). The 50 most downregulated and 50 most upregulated mRNAs in nonAC-hMSCs at both time points are shown as a heat map in Fig. 1a and are listed in Table 1. The expression of VEGFA in the nonAC-hMSCs was significantly higher than that in the AC-hMSCs at the two time points (Table 1). This increase in VEGFA mRNA in the nonAChMSCs was also verified by RT-qPCR. Western blot results showed that the VEGFA protein level was higher in nonAC-hMSCs than in AC-hMSCs (Fig. 1c, d; Additional file 4: Figure S2A). Moreover, the secretion of VEGFA, as determined by ELISA, was significantly higher in nonAC-hMSCs than in AC-hMSCs (Fig. 1e). Taken together, these results indicate that the nonadherent culture method elevates the expression of VEGFA in hMSCs and facilitates VEGFA secretion.

\section{Decreased levels of VEGFA produced by VEGFA knockdown nonAC-hMSCs inhibit the angiogenesis of HUVECS}

A lentivirus-based system was used to assess whether the expression of VEGFA in hMSCs plays an important role in HUVEC angiogenesis. VEGFA was stably overexpressed in AC-hMSCs (AC-VEGFA-hMSCs) and depleted in nonAC-hMSCs (nonAC-shVEGFA-hMSCs) (Fig. 2a, b; Additional file 4: Figure S2B). ELISA analysis revealed that forced expression of VEGFA induced VEGFA production, whereas knockdown of VEGFA led to the converse (Fig. 2c). We performed a tube formation assay to detect angiogenesis of HUVECs and found that HUVECs cultured in CM from AC-VEGFA-hMSCs and nonAC-hMSCs formed well-developed networks of capillary-like tubes. In contrast, HUVECs cultured in 
A

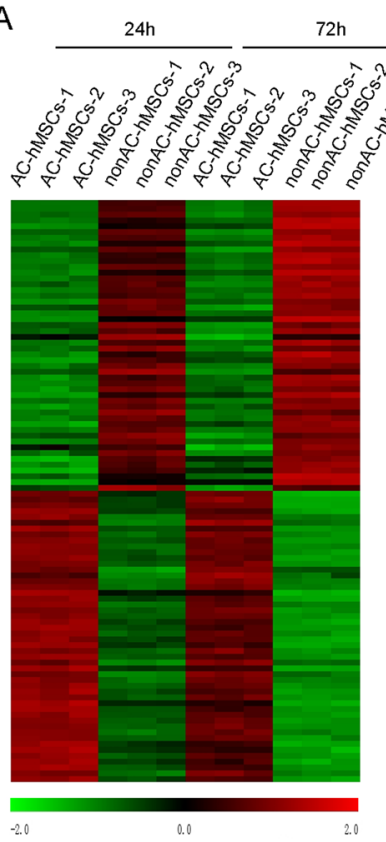

B

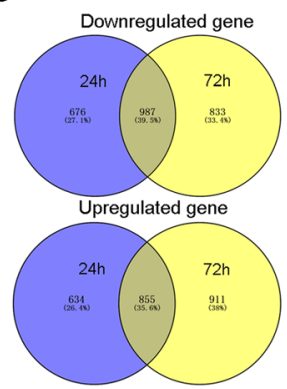

D

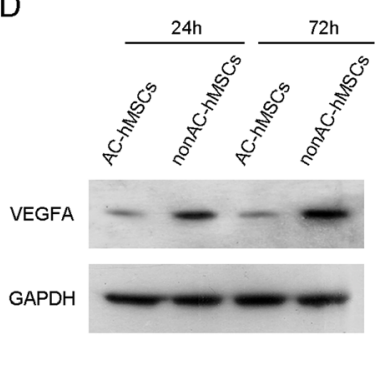

C

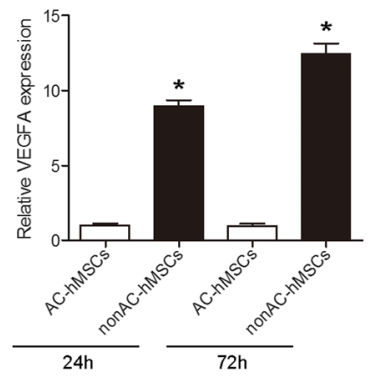

E

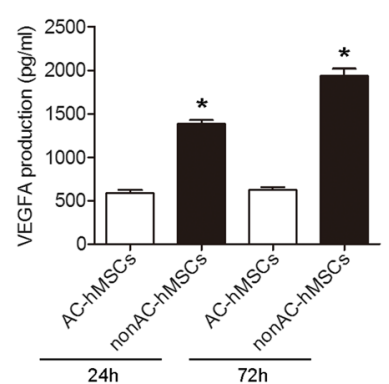

Fig. 1 Increased VEGFA expression is observed in the nonAC-hMSCs at two different time points. a The 50 most downregulated and 50 most upregulated mRNAs in the AC-hMSCs and nonAC-hMSCs at $24 \mathrm{~h}$ and $72 \mathrm{~h}$. $\mathbf{b}$ Venn diagram of upregulated and downregulated genes in the AChMSCs and nonAC-hMSCs at $24 \mathrm{~h}$ and $72 \mathrm{~h}$. c VEGFA mRNA and $\mathbf{d}$ protein expression levels in AC-hMSCs and nonAC-hMSCs at $24 \mathrm{~h}$ and $72 \mathrm{~h}$ were measured by RT-qPCR and western blotting, respectively. e VEGFA secretion from hMSCs as determined by ELISA. Data are represented as mean \pm SD ( $n=3$ per group). ${ }^{*} P<0.05$

CM from nonAC-shVEGFA-hMSCs and AC-hMSCs displayed very few capillary-like structures (Fig. 2d). Collectively, these data suggest that silencing and therefore limiting secretion of VEGFA in nonAC-hMSCs hindered tube formation of HUVECs, whereas excess VEGFA secretion in AC-hMSCs promoted HUVEC tube formation.

\section{VEGFA production by VEGFA-overexpressing AC-hMSCs} increases angiogenesis and reduces infarction size after MI

To explore the functional significance of VEGFA in MSC-mediated angiogenesis in a rat MI model, we transplanted AC-VEGFA-hMSCs, nonAC-shVEGFAhMSCs, or their corresponding control cells into the ischemic LV wall border zone and analyzed VEGFA expression and secretion after cell transplantation. Compared to the corresponding control groups, ACVEGFA-hMSC-transplanted group and nonAC-hMSCtransplanted group had significantly higher levels of VEGFA expression and secretion, whereas nonACshVEGFA-hMSC-transplanted group and AC-hMSCtransplanted group exhibited significantly lower levels of VEGFA expression and secretion (Fig. 3a-c; Additional file 4: Figure S2C). Furthermore, we evaluated angiogenesis in the infarcted heart after hMSC transplantation using immunohistochemistry and found that the number of vWF-positive vessels significantly increased in ACVEGFA-hMSC- and nonAC-hMSC-treated groups, while they were markedly decreased in nonAC-shVEGFAhMSC- and AC-hMSC-treated groups (Fig. 3d). Additionally, we used Masson's trichrome staining to detect the extent of fibrosis after hMSC transplantation. The results showed that the infarct size was significantly smaller in the AC-VEGFA-hMSC-injected hearts and nonAChMSC-injected hearts than in the control AC-hMSCinjected hearts, whereas it was significantly larger in the nonAC-shVEGFA-hMSC-injected hearts and AC-hMSCinjected hearts than in the control nonAC-hMSC-injected hearts (Fig. 3e). Therefore, our data demonstrate that implantation of AC-VEGFA-hMSCs promotes angiogenesis and decreases infarction size by stimulating VEGFA production in the rat MI model.

\section{VEGFA secretion by VEGFA-overexpressing AC-hMSCs improves cardiac functions in a rat $\mathrm{MI}$ model}

Echocardiography was performed 4 weeks after hMSC implantation in a rat MI model to assess the therapeutic effects of MSCs on cardiac function (Fig. 4a). LVEDD and LVESD were significantly lower in the AC-VEGFAhMSC and nonAC-hMSC groups and significantly higher in the nonAC-shVEGFA-hMSC and AC-hMSC groups compared to the corresponding control groups 
Table 1 Differential expression of genes in AC-hMSCs and nonAC-hMSCs at two different time points

\begin{tabular}{|c|c|c|c|c|c|c|}
\hline \multirow{2}{*}{$\begin{array}{l}\text { Gene } \\
\text { name }\end{array}$} & \multicolumn{3}{|l|}{$24 \mathrm{~h}$} & \multicolumn{3}{|l|}{$72 \mathrm{~h}$} \\
\hline & Fold change & $p$ value & Regulation & Fold change & $p$ value & Regulation \\
\hline C2CD4B & 111.8401912 & 0.000145643 & Up & 1340.34 & 0.000682199 & Up \\
\hline SSTR2 & 48.09021308 & 0.000555557 & Up & 307.6615 & 0.000223379 & Up \\
\hline SLC16A6 & 58.22005835 & 0.000188778 & Up & 244.5447 & 0.0005466866 & Up \\
\hline FAM65C & 19.69493511 & $2.3287 \mathrm{E}-08$ & Up & 213.9348 & 3.43255E-05 & Up \\
\hline WDR86 & 29.35821269 & 2.03996E-05 & Up & 190.5289 & $6.26129 \mathrm{E}-05$ & Up \\
\hline GJB2 & 33.73311298 & 2.37009E-05 & Up & 170.4077 & 0.000560036 & Up \\
\hline CXCR4 & 36.63486255 & 0.000412617 & Up & 156.6291 & 0.000381246 & Up \\
\hline MMP13 & 18.1969967 & 0.001865303 & Up & 119.5122 & $1.05064 \mathrm{E}-07$ & Up \\
\hline BMP2 & 20.03644612 & 0.000193438 & Up & 117.9299 & $2.56801 \mathrm{E}-05$ & Up \\
\hline C2CD4A & 14.64162866 & 0.0061597 & Up & 85.52482 & 0.000625896 & Up \\
\hline CLEC2B & 13.20455766 & 0.000159395 & Up & 78.42479 & 0.002468774 & Up \\
\hline TNFSF10 & 29.04491724 & 0.001267072 & Up & 57.67481 & 0.000386508 & Up \\
\hline PRSS35 & 11.75846586 & 0.000587899 & Up & 56.35998 & 0.000564307 & Up \\
\hline MEGF10 & 21.16086411 & 0.002528035 & Up & 55.27018 & 5.18695E-05 & Up \\
\hline DUSP4 & 13.21396805 & 9.34513E-05 & Up & 54.87385 & 0.001212616 & Up \\
\hline TSPAN11 & 27.83430153 & 0.000297067 & Up & 53.68215 & $9.89532 \mathrm{E}-07$ & Up \\
\hline DRAXIN & 13.45155294 & 0.000251701 & Up & 51.35024 & 0.000127201 & Up \\
\hline PDK4 & 96.98929796 & 5.63176E-05 & Up & 46.77971 & 2.99919E-05 & Up \\
\hline ANKH & 10.78789239 & 0.004008663 & Up & 46.26112 & $2.57026 \mathrm{E}-05$ & Up \\
\hline RGS16 & 39.28019753 & 0.000545809 & Up & 45.46778 & 7.36326E-07 & Up \\
\hline RANBP3L & 9.229649839 & 5.07326E-06 & Up & 38.70846 & 7.23795E-05 & Up \\
\hline ITGA2 & 21.59037248 & 5.13189E-05 & Up & 38.41117 & 0.000872801 & Up \\
\hline ADGRE2 & 9.283517607 & 0.008575709 & Up & 34.87146 & $6.97014 \mathrm{E}-06$ & Up \\
\hline MMP11 & 16.87338081 & 0.00285751 & Up & 34.62489 & 0.001476287 & Up \\
\hline KLRK1 & 11.95483946 & 0.000405053 & Up & 34.56032 & 0.002009842 & Up \\
\hline BEGAIN & 33.51924802 & $6.25637 \mathrm{E}-07$ & Up & 30.53104 & 0.000704421 & Up \\
\hline$P P L$ & 9.869982413 & 0.009031407 & Up & 25.65778 & 0.000467585 & Up \\
\hline $\mathrm{ABCG} 1$ & 30.41288703 & 0.000525295 & Up & 24.69536 & $4.16571 \mathrm{E}-07$ & Up \\
\hline CITED1 & 18.69977736 & 0.000106336 & Up & 24.55424 & 0.000276694 & Up \\
\hline SYTL5 & 36.85560246 & 0.000548581 & Up & 23.16011 & 0.001025661 & Up \\
\hline RGS17 & 12.57031107 & 0.000155551 & Up & 22.45184 & 0.000241119 & Up \\
\hline $\mathrm{KIAA} 1211 \mathrm{~L}$ & 16.30985794 & $1.57458 \mathrm{E}-05$ & Up & 22.31267 & 3.31962E-05 & Up \\
\hline GLDN & 38.48393676 & 0.000844764 & Up & 21.32752 & 0.000790957 & Up \\
\hline EXPH5 & 15.81689248 & 0.001356464 & Up & 20.98551 & 0.001995397 & Up \\
\hline PTGDR & 14.69735545 & 0.002868926 & Up & 18.19271 & 0.000615557 & Up \\
\hline CCR1 & 19.99097768 & 7.95976E-05 & Up & 16.40048 & 0.002030008 & Up \\
\hline RASSF10 & 32.93415505 & 0.002178622 & Up & 16.05662 & 0.000741795 & Up \\
\hline FAM189A1 & 11.84572522 & 0.005621653 & Up & 15.56768 & $1.4842 \mathrm{E}-06$ & Up \\
\hline COLEC12 & 23.05965011 & 0.000724925 & Up & 15.25867 & 0.00408674 & Up \\
\hline KLRC1 & 10.05484612 & 0.002881202 & Up & 15.20619 & 0.000154447 & Up \\
\hline VEGFA & 9.752636526 & 4.13371E-06 & Up & 14.73218 & 0.000346357 & Up \\
\hline XKRX & 16.61076595 & 0.000501039 & Up & 14.38702 & 0.000172706 & Up \\
\hline RASGRP3 & 2.106425558 & 0.028207257 & Up & 13.9441 & 7.03918E-05 & Up \\
\hline
\end{tabular}


Table 1 Differential expression of genes in AC-hMSCs and nonAC-hMSCs at two different time points (Continued)

\begin{tabular}{|c|c|c|c|c|c|c|}
\hline \multirow{2}{*}{$\begin{array}{l}\text { Gene } \\
\text { name }\end{array}$} & \multicolumn{3}{|l|}{$24 \mathrm{~h}$} & \multicolumn{3}{|l|}{$72 \mathrm{~h}$} \\
\hline & Fold change & $p$ value & Regulation & Fold change & $p$ value & Regulation \\
\hline ENTPD3 & 9.912879304 & 0.001624712 & Up & 13.76377 & 0.000782473 & Up \\
\hline YPEL4 & 53.61892782 & $3.5446 \mathrm{E}-05$ & Up & 13.63856 & 0.002333755 & Up \\
\hline SLC7A8 & 11.49043722 & 0.011671519 & Up & 13.22151 & 0.002134691 & Up \\
\hline $\mathrm{CH} 25 \mathrm{H}$ & 30.44303155 & 0.001762942 & Up & 12.42755 & 0.004566846 & Up \\
\hline CYFIP2 & 2.709902602 & 0.007297695 & Up & 10.76685 & $8.22518 \mathrm{E}-05$ & Up \\
\hline C3AR1 & 8.089947094 & 0.000411377 & Up & 9.559041 & 0.000110391 & Up \\
\hline F2RL1 & 8.771762767 & 0.000415707 & Up & 9.21932 & 0.000385279 & Up \\
\hline CPA4 & 0.060443451 & 0.004894715 & Down & 0.005735 & $3.23423 \mathrm{E}-05$ & Down \\
\hline CNN1 & 0.071987351 & 0.002896126 & Down & 0.005827 & 0.001199281 & Down \\
\hline MEST & 0.041677656 & 0.002021602 & Down & 0.006979 & $1.73885 \mathrm{E}-05$ & Down \\
\hline KRT34 & 0.011913749 & 0.00081183 & Down & 0.007354 & $5.15434 \mathrm{E}-05$ & Down \\
\hline DKK1 & 0.004639044 & 0.000883335 & Down & 0.010773 & $4.20121 \mathrm{E}-05$ & Down \\
\hline LMCD1 & 0.029802085 & $2.33205 E-05$ & Down & 0.012196 & 0.0006374 & Down \\
\hline KRTAP2-3 & 0.011067704 & 0.000539452 & Down & 0.015092 & 0.000257229 & Down \\
\hline FAM46B & 0.033933237 & 4.34704E-06 & Down & 0.0174 & 0.00090771 & Down \\
\hline MYBL1 & 0.029145858 & 1.44931E-05 & Down & 0.017623 & 0.001442927 & Down \\
\hline NEIL3 & 0.017315518 & 0.000108138 & Down & 0.01963 & 0.00027453 & Down \\
\hline KRTAP1-5 & 0.029877632 & 0.001686929 & Down & 0.019696 & 0.000129879 & Down \\
\hline SPC25 & 0.044301408 & 0.001309748 & Down & 0.027418 & 0.001322887 & Down \\
\hline THBS1 & 0.055229076 & 0.000388028 & Down & 0.028426 & 0.002471628 & Down \\
\hline PTX3 & 0.010465082 & 0.001271904 & Down & 0.028936 & 3.57597E-05 & Down \\
\hline TSPAN18 & 0.053314954 & 0.000181383 & Down & 0.029322 & 0.007596185 & Down \\
\hline KRTAP2-2 & 0.048512804 & $1.88464 \mathrm{E}-05$ & Down & 0.029763 & 0.00057951 & Down \\
\hline MGAM & 0.034235468 & 0.00061814 & Down & 0.030484 & 0.001303756 & Down \\
\hline ANLN & 0.05142361 & 0.001527223 & Down & 0.031571 & 0.001433314 & Down \\
\hline KIRREL3 & 0.057630141 & 0.002929558 & Down & 0.03209 & 0.000359608 & Down \\
\hline E2F8 & 0.018025232 & $1.75885 \mathrm{E}-05$ & Down & 0.032301 & 0.000149989 & Down \\
\hline CEP55 & 0.0606109 & 0.000871839 & Down & 0.033875 & 0.000278821 & Down \\
\hline CTGF & 0.048584673 & 0.000553306 & Down & 0.034772 & 0.000177803 & Down \\
\hline SKA1 & 0.04626685 & 1.04503E-05 & Down & 0.034872 & 0.000282226 & Down \\
\hline KRTAP1-1 & 0.029512423 & 2.68546E-06 & Down & 0.036294 & 0.001195564 & Down \\
\hline KRTAP1-4 & 0.034637793 & 8.67165E-05 & Down & 0.032924 & 0.000393189 & Down \\
\hline SHCBP1 & 0.074874368 & 0.007927709 & Down & 0.037302 & 4.80244E-05 & Down \\
\hline CDC45 & 0.048785792 & 0.001757321 & Down & 0.038191 & 0.002567892 & Down \\
\hline MKI67 & 0.061895471 & 0.001531656 & Down & 0.039185 & 0.006627038 & Down \\
\hline ERCC6L & 0.031855904 & $1.35451 \mathrm{E}-06$ & Down & 0.040946 & 0.001070963 & Down \\
\hline TNFRSF11B & 0.035805553 & 0.004205691 & Down & 0.041701 & 0.000357158 & Down \\
\hline ZNF367 & 0.048382669 & 0.003338013 & Down & 0.042294 & 8.8958E-05 & Down \\
\hline DMD & 0.038750757 & $1.1526 \mathrm{E}-05$ & Down & 0.042472 & 0.001858382 & Down \\
\hline CDCA8 & 0.038925122 & 0.000236484 & Down & 0.043372 & 0.000204384 & Down \\
\hline PADI1 & 0.041441366 & 0.000851724 & Down & 0.044515 & 7.47813E-06 & Down \\
\hline HIST2H3C & 0.045817546 & 0.00054878 & Down & 0.045124 & 0.000144333 & Down \\
\hline TTK & 0.061307423 & $7.72508 \mathrm{E}-05$ & Down & 0.045391 & 0.000936671 & Down \\
\hline
\end{tabular}


Table 1 Differential expression of genes in AC-hMSCs and nonAC-hMSCs at two different time points (Continued)

\begin{tabular}{|c|c|c|c|c|c|c|}
\hline \multirow{2}{*}{$\begin{array}{l}\text { Gene } \\
\text { name }\end{array}$} & \multicolumn{3}{|l|}{$24 \mathrm{~h}$} & \multicolumn{3}{|l|}{$72 \mathrm{~h}$} \\
\hline & Fold change & $p$ value & Regulation & Fold change & $p$ value & Regulation \\
\hline GABBR2 & 0.045546263 & 0.001708625 & Down & 0.045807 & $9.62305 \mathrm{E}-05$ & Down \\
\hline FAM111B & 0.027357567 & 4.93311E-06 & Down & 0.046582 & 0.000558007 & Down \\
\hline CCIN & 0.051329319 & 0.003246805 & Down & 0.048819 & 5.93123E-05 & Down \\
\hline KIF15 & 0.066141532 & 0.000117669 & Down & 0.04886 & 0.001136135 & Down \\
\hline NCKAP5 & 0.066816867 & 0.000303257 & Down & 0.049423 & 0.001324705 & Down \\
\hline NTF3 & 0.042448723 & 0.001215131 & Down & 0.050437 & 0.005324032 & Down \\
\hline UBE2C & 0.065284894 & 4.29692E-06 & Down & 0.052099 & 0.002768879 & Down \\
\hline KRT33B & 0.064358986 & 0.00039797 & Down & 0.052188 & 0.000124335 & Down \\
\hline MCM10 & 0.046540673 & 0.000257847 & Down & 0.054035 & 0.003217037 & Down \\
\hline LMOD1 & 0.054276425 & 1.14094E-06 & Down & 0.055313 & 0.000663673 & Down \\
\hline LTF & 0.068482765 & 1.6193E-05 & Down & 0.057596 & 0.000575741 & Down \\
\hline HIST1H3G & 0.035683104 & 0.000992525 & Down & 0.058962 & 0.000146762 & Down \\
\hline CCDC85A & 0.066799698 & 9.312090059 & Down & 0.059079 & 0.009067666 & Down \\
\hline NCAPG & 0.073153137 & 6.164797229 & Down & 0.060212 & 0.037036192 & Down \\
\hline
\end{tabular}
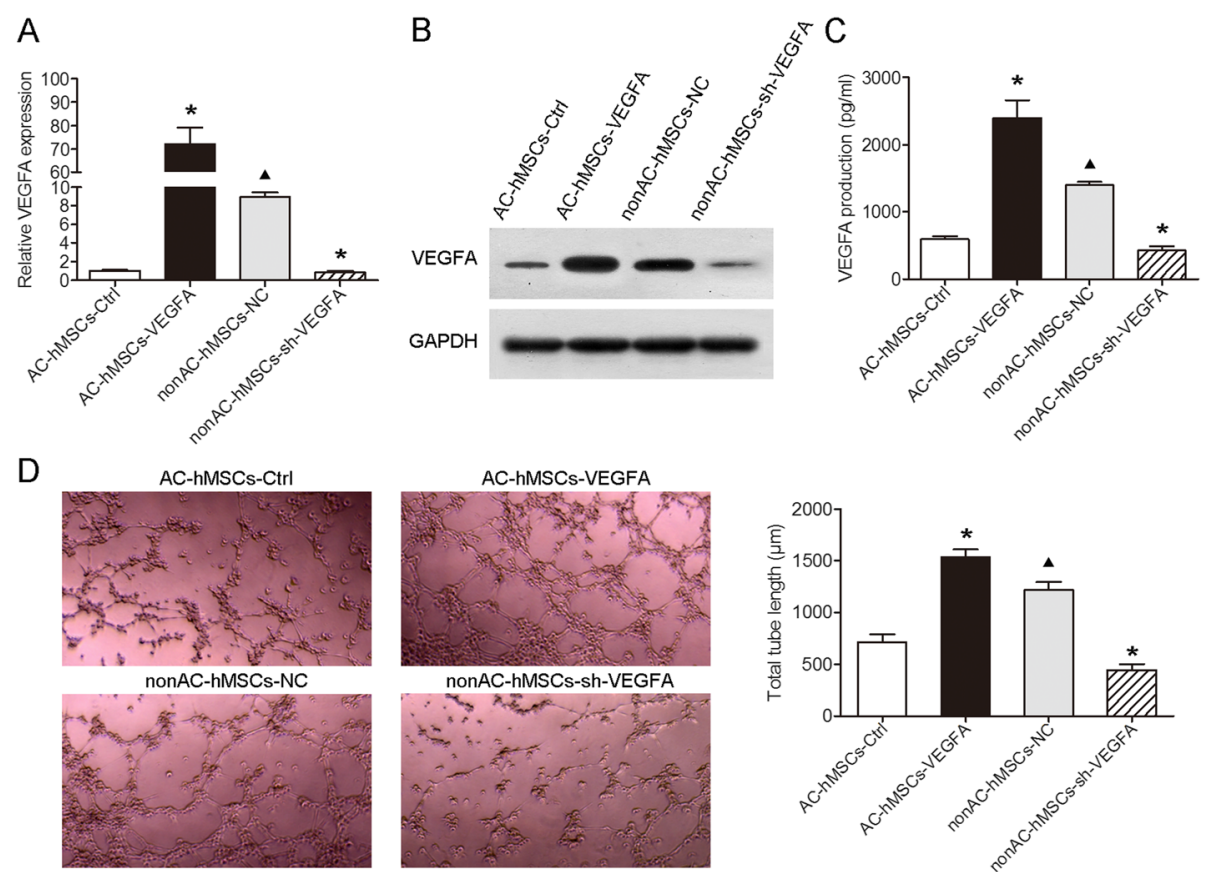

Fig. 2 Decreased levels of VEGFA produced by VEGFA knockdown nonAC-hMSCs inhibits the angiogenesis of HUVECs. AC-hMSCs were infected with VEGFA or negative control (NC) lentiviruses and nonAC-hMSCs were infected with shVEGFA or the control lentiviruses. $\mathbf{a}$ RT-qPCR and $\mathbf{b}$ western blotting were performed to detect mRNA and protein levels of VEGFA, respectively. c ELISA analysis was performed to determine the levels of secreted VEGF. $\mathbf{d}$ Capillary-like structure formation of HUVECs cultured in CM from hMSCs with different genotypes was evaluated by tube formation assay. Data are represented as mean \pm SD ( $n=3$ per group). ${ }^{*} P<0.05$ compared to AC-hMSC-Ctrl or nonAC-hMSC-NC group. $\boldsymbol{\Delta} P<0.05$ compared to AC-hMSC-Ctrl group 


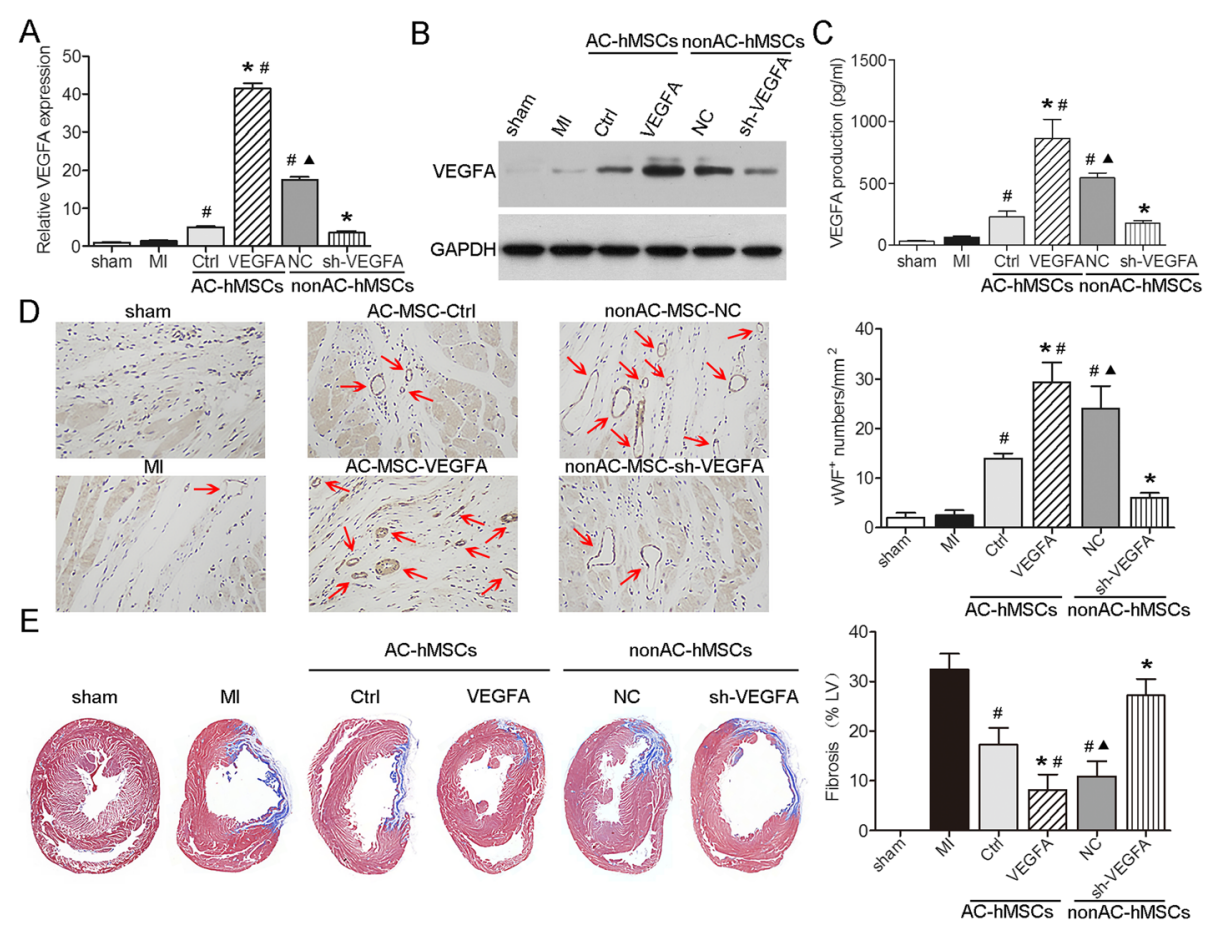

Fig. 3 VEGFA production by VEGFA-overexpressing AC-hMSCs increases angiogenesis and reduces infarction size after MI. a RT-qPCR, b western blotting, and $\mathbf{c}$ ELISA were used to assess VEGFA expression and secretion in the heart tissues obtained from LADCA-ligated rats $48 \mathrm{~h}$ after transplantation with AC-VEGFA-hMSCs, nonAC-sh-VEGFA-hMSCs, or their corresponding control hMSCs. $\mathbf{d}$ Immunohistochemistry analysis for vWF expression in the heart tissues obtained from LADCA-ligated rats 28 days after transplantation with AC-VEGFA-hMSCs, nonAC-sh-VEGFA-h MSCs, or their corresponding control hMSCs. Red arrow indicates the small vessels. e Representative images and quantification of fibrosis area. Data are represented as mean \pm SD ( $n=8$ per group). ${ }^{*} P<0.05$ compared to AC-hMSC-Ctrl or nonAC-hMSC-NC group. ${ }^{*} P<0.05$ compared to Ml group. $\boldsymbol{\Delta} P<0.05$ compared to AC-hMSC-Ctrl group

(Fig. 4b, c). Furthermore, the AC-VEGFA-hMSC and nonAC-hMSC groups exhibited significantly increased LVEF and LVFS, whereas the nonAC-shVEGFA-hMSC and AC-hMSC groups displayed significantly decreased LVEF and LVFS (Fig. 4d, e). Collectively, these findings demonstrate that implantation of VEGFA-overexpressing AC-hMSCs could decrease myocardial remodeling.

\section{VEGFA is a direct target of miR-519d}

To elucidate the molecular mechanism of VEGFA in the regulation of angiogenesis, we first employed the online bioinformatics (TargetScan and microrna.org) to predict the potential microRNA (miRNA) binding sites in VEGFA. miR-519d was found to be a putative miRNA for the regulation of VEGFA (Fig. 5a). We then probed for miR-519d expression in AC-hMSCs and nonAC-hMSCs at two different time points using RT-qPCR and found that miR-519d expression was significantly lower in nonAC-hMSCs compared to AC-hMSCs (Fig. 5b). As shown in Fig. 1c, d, the expression of VEGFA was significantly higher in nonAChMSCs than in AC-hMSCs. This negative correlation between VEGFA and miR-519d expression indicates that miR-519d may be involved in the regulation of VEGFA.
To validate a direct interaction between VEGFA and miR-519d, a luciferase reporter plasmid containing wildtype or mutant VEGFA 3' UTR was constructed and transfected into HEK293T cells, along with miR-519d or control mimics. Results showed that miR-519d led to a significant reduction in the luciferase activity produced by the construct containing the VEGFA putative miR-519d binding site, whereas miR-519d had no significant effect on the construct containing the mutant VEGFA 3' UTR (Fig. 5c). Furthermore, RT-qPCR, western blot, and ELISA showed that overexpression of miR-519d in nonAC-hMSCs significantly decreased the expression and secretion of VEGFA, and knockdown of miR-519d in AC-hMSCs enhanced VEGFA expression and secretion (Fig. 5d-f; Additional file 4: Figure S2D). Taken together, these results indicate that miR-519d exerts suppressive effects on VEGFA expression by binding the VEGFA 3' UTR.

The secretion of VEGFA in VEGFA-overexpressing nonAChMSCs attenuates miR-519d-mediated inhibition of tube formation of HUVECs

We performed a rescue experiment to determine whether miR-519d regulates cell angiogenesis via 

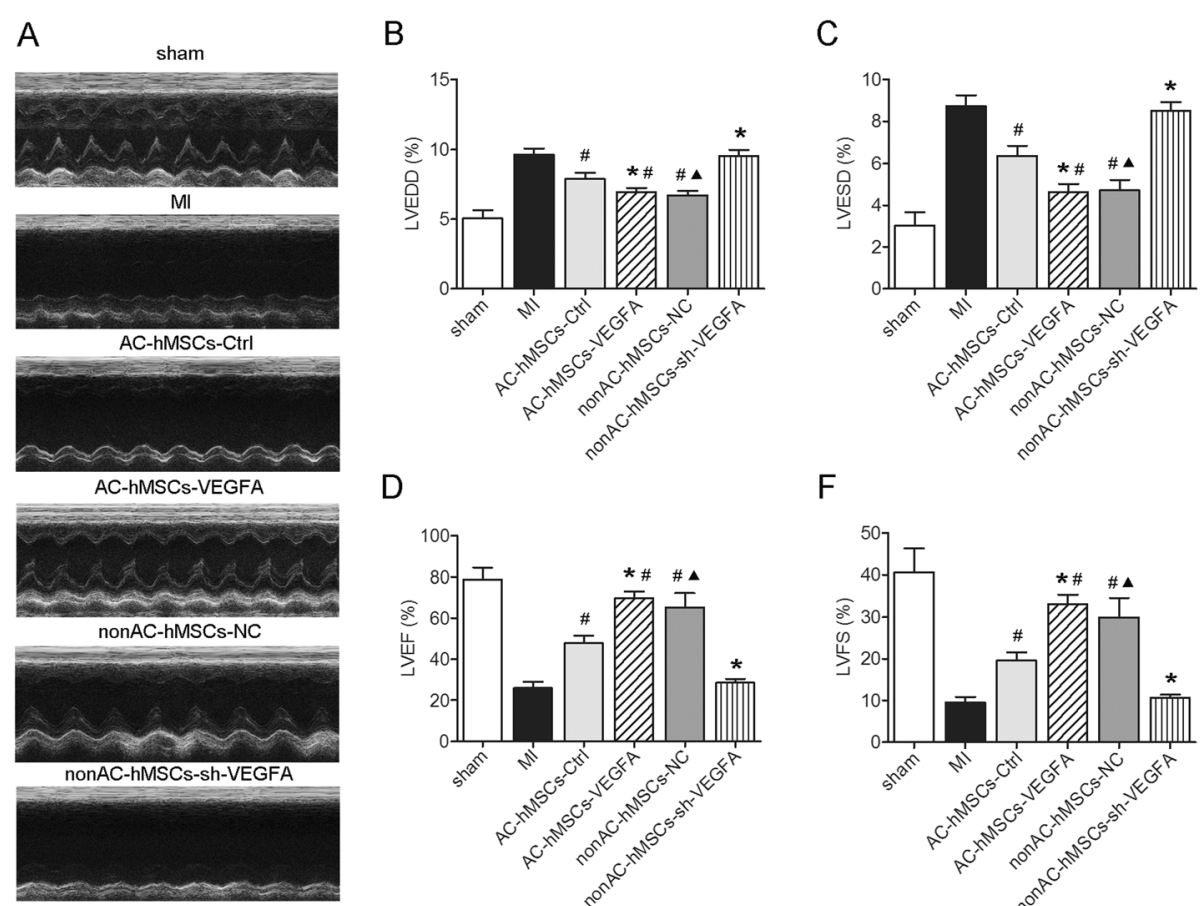

D

$\mathrm{F}$
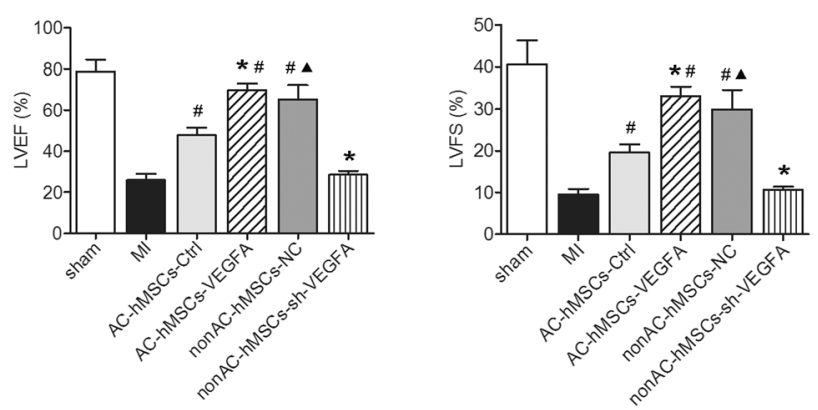

Fig. 4 VEGFA secretion by VEGFA-overexpressing AC-hMSCs improves cardiac functions in a rat Ml model. a Cardiac function was determined by echocardiography 28 days after MI induction and hMSC transplantation. b Quantification of LVEDD, $\mathbf{c}$ LVESD, $\mathbf{d}$ LVEF, and e LVFS. Data are represented as mean \pm SD ( $n=8$ per group). ${ }^{*} P<0.05$ compared to AC-hMSC-Ctrl or nonAC-hMSC-NC group. ${ }^{\#} P<0.05$ compared to Ml group. ${ }^{\mathbf{\Delta}} P<0.05$ compared to AC-hMSC-Ctrl group

targeting VEGFA. nonAC-hMSCs were co-transfected with miR-519d mimics and VEGFA plasmids for $24 \mathrm{~h}$. RT-qPCR and western blot analyses indicated that ectopic expression of VEGFA attenuated miR-519dmediated suppression of VEGFA expression (Fig. 6a, b; Additional file 4: Figure S2E) and secretion (Fig. 6c). Furthermore, while miR-519d-overexpressing decreased tube formation of HUVECs, the addition of VEGFA derived from VEGFA-overexpressing nonAChMSCs reversed this effect (Fig. 6d). Collectively, these data suggest that miR-519d exerts its biological effects by directly targeting VEGFA.

\section{Discussion}

A growing body of evidence suggests that the beneficial therapeutic effects of MSCs are the result of paracrine mechanisms [26, 27]. Various growth factors, cytokines, and angiogenic factors released from MSCs play critical roles in improving the function of infracted hearts [28, 29]. VEGFA belongs to the VEGF family and is a wellknown pro-angiogenic factor. VEGFA has been reported to stimulate endothelial cell proliferation, migration, and tube formation, thereby promoting angiogenesis [30]. Recent studies indicated that VEGFA released from different types of stem cells, including MSCs, have the ability to treat various human diseases, such as myocardial ischemia, by regulation of angiogenesis [31-33]. Markel et al. [31] reported that VEGFA knockdown in MSCs impaired stem cell-mediated myocardial function. Cho et al. [32] reported that VEGFA-secreting human umbilical cord blood-derived MSCs decreased infarct size and improved cardiac function via enhancing angiogenesis in infarct myocardium. Kim et al. [23] also reported that the transplantation of hypoxia inducible VEGFAexpressing MSCs promoted ischemia-responsive VEGFA production, resulting in a significant decrease in apoptosis and a significant increase in micro-vessel formation after MI. In our study, VEGFA was upregulated in nonAC-hMSCs (Fig. 1). The high levels of VEGFA secreted by AC-VEGFA-hMSCs facilitated HUVEC angiogenesis, while the low expression levels of VEGFA produced by nonAC-shVEGFA-hMSCs suppressed this process (Fig. 2). In a rat MI model, administration of AC-VEGFA-hMSCs significantly increased VEGFA production, leading to improvement in myocardial function, enhancement in vessel density, and reduction in infarct size, whereas reversed results were observed in nonACshVEGFA-hMSC-transplanted hearts (Figs. 3 and 4). Recently, it has been reported that VEGFA secretion protects cardiomyocytes against ischemia [33]. Additionally, VEGF is known to modulate macrophage recruitment and macrophage phenotype by promoting $\mathrm{M} 1 \geq \mathrm{M} 2$ 


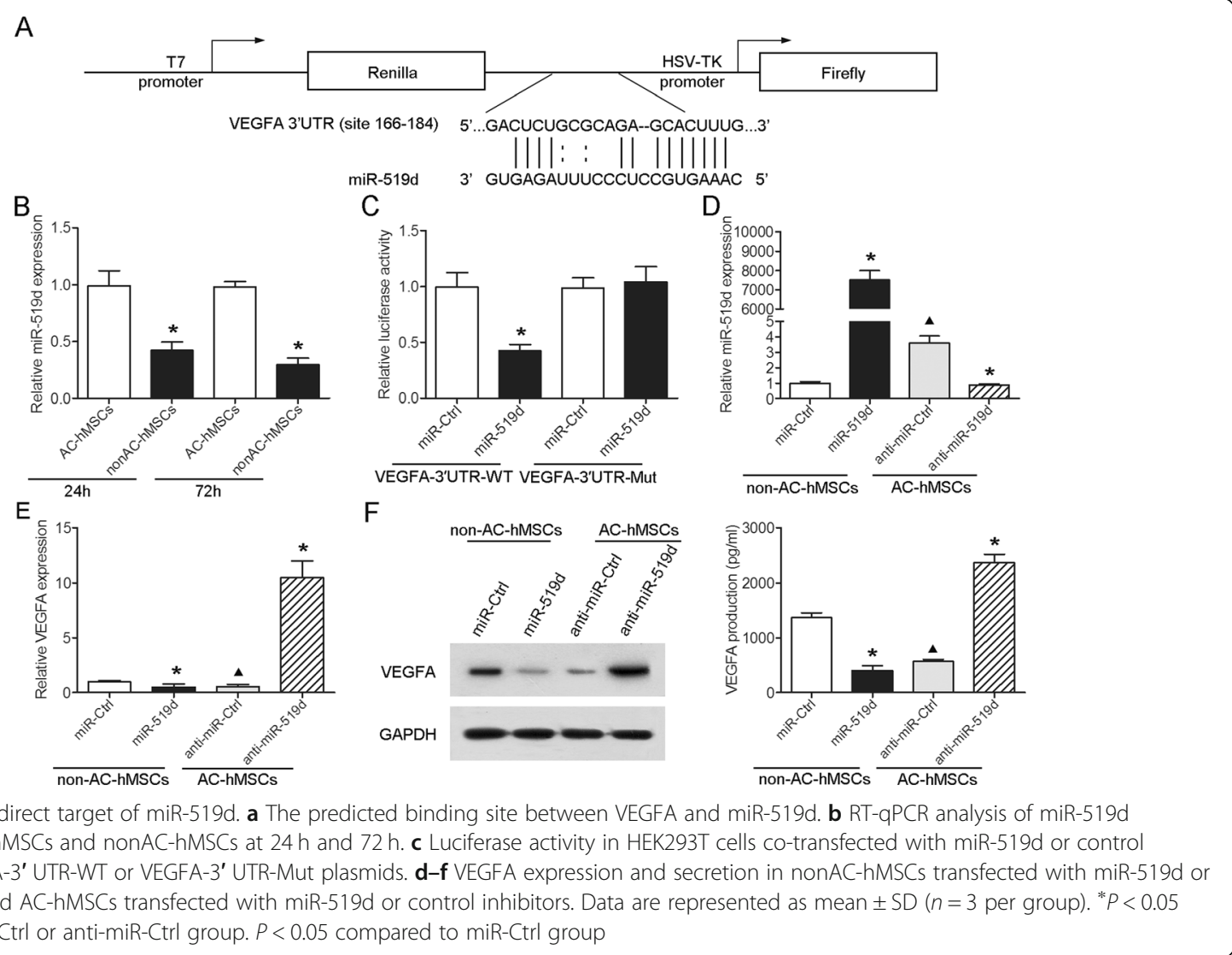

transpolarization [34, 35]. In skin cancer, VEGFA promotes macrophage recruitment to the tumor and thereby facilitates tumor-associated macrophage development [34]. In chronic kidney disease, ELP-VEGF therapy distinctly shifted renal macrophage phenotype from proinflammatory M1 to VEGF expressing M2, restoring VEGF signaling and sustaining improvement of renal function and microvascular integrity [35]. However, whether VEGFA improves cardiac function by affecting cardiomyocytes or regulating macrophage recruitment and macrophage phenotype needs further investigation.

A number of studies have indicated that VEGFA can be directly regulated by miRNAs $[36,37]$. miR-299-3p inhibited cell proliferation and motility and induced apoptosis in renal cell carcinoma by directly targeting VEGFA [36]. miR-199a-5p impaired both the survival and angiogenic capacity of MSCs by directly regulating VEGFA expression [37]. In our study, miR-519d directly affected VEGFA expression (Fig. 5). miR-519d is one member of the chromosome 19 miRNA cluster, which is the largest human miRNA cluster containing 46 premiRNAs [38]. There is growing evidence showing that miR-519d is involved in a range of biological processes, including cell proliferation, apoptosis, differentiation, migration, and angiogenesis [39-42]. miR-519d overexpression facilitated the proliferation, migration, invasion, and adhesion of melanoma cells in vitro and accelerated lung metastatic capability in vivo [39]. Expression of miR-519d promoted hepatocellular carcinoma cell proliferation and invasion and inhibited apoptosis [40]. On the other hand, overexpression of miR-519d impaired colorectal cancer cell viability, migration, and invasion and induced G0/G1 phase arrest and apoptosis by downregulating TROAP expression [41]. Additionally, miR-519d expression attenuated endothelial cell growth, migration, and tube formation [42]. In this study, increased VEGFA production in nonAC-hMSCs reversed the suppressive effect of miR519d on HUVEC tube formation (Fig. 6).

How does the nonadherent culture method regulate the expression of miR-519d? Our previous research showed that the nonadherent culture method decreased Sca-1 expression [14]. Our microarray analysis also demonstrated that the nonadherent culture method upregulated CXCR4 expression. Based on these data, we hypothesized that the nonadherent culture method might regulate the expression of miR-519d by regulating the adhesion molecules of MSCs. Further investigation is needed to verify this hypothesis. 


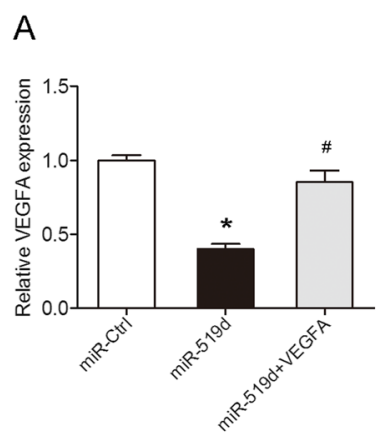

B

C

D
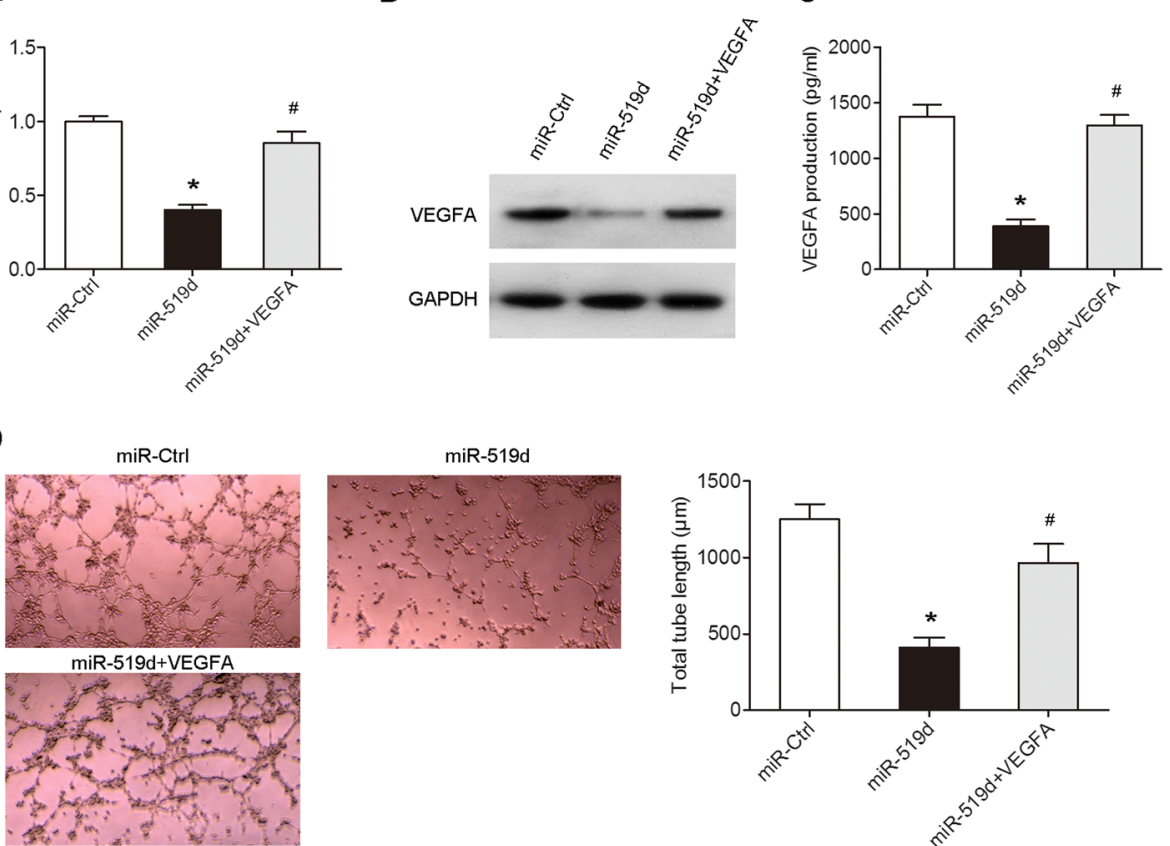

miR-519d
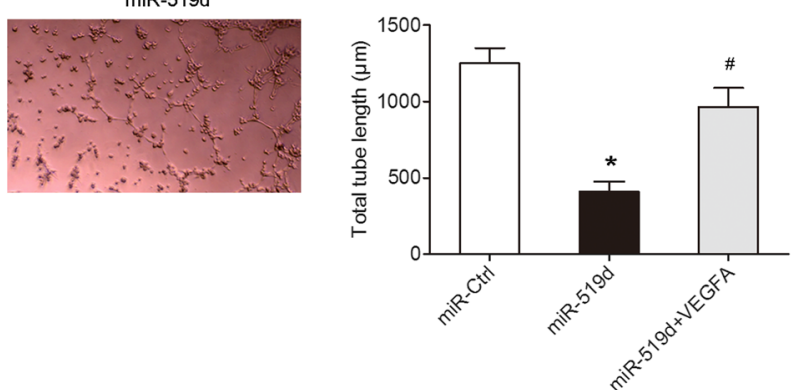

Fig. 6 The secretion of VEGFA in VEGFA-overexpressing nonAC-hMSCs attenuates miR-519d-mediated inhibition of tube formation of HUVECs. a The expression and secretion of VEGFA in the nonAC-hMSCs co-transfected with miR-519d mimics and VEGFA plasmid were measured using RT-qPCR, $\mathbf{b}$ western blotting, and $\mathbf{c}$ ELISA, respectively. $\mathbf{d}$ Tube formation assay was performed to detect the capillary-like tubules formation of HUVECs cultured in the presence of hMSC-conditioned medium. Data are represented as mean \pm SD ( $n=3$ per group). ${ }^{*} P<0.05$ compared to miR-Ctrl group. ${ }^{\#} P<0.05$ compared to miR-519d group

\section{Conclusions}

In summary, we determined that the nonadherent culture of MSCs increased the secretion of VEGFA, directly opposing the effects of miR-519d (Additional file 5: Figure S3). VEGFA promoted the tube formation capabilities of HUVECs in vitro, decreased myocardial remodeling and infarct size, and increased angiogenesis in a rat MI model. These findings provide a novel experimental protocol to optimize MSCbased therapies for the treatment of MI.

\section{Supplementary information}

Supplementary information accompanies this paper at https://doi.org/10. 1186/s13287-020-01780-x.

Additional file 1 : Figure S1. Characterization of isolated hMSCs. Flow cytometry showing the percentage of CD73, CD90, CD105, CD11b, CD14, CD34 and CD45 hMSCs.

Additional file 2 : Table S1. Differential expression of genes in AChMSCs and nonAC-hMSCs at $24 \mathrm{~h}$.

Additional file $\mathbf{3}$ : Table S2. Differential expression of genes in AChMSCs and nonAC-hMSCs at $72 \mathrm{~h}$.

Additional file 4 : Figure S2. Relative VEGFA protein expression in the different groups. Data are represented as mean \pm SD $(n=3$ per group). ${ }^{*} P<0.05$ compared to AC-hMSCs, AC-hMSCs-Ctrl, nonAC-hMSCsNC, miR-Ctrl or anti-miR-Ctrl group. ${ }^{\#} P<0.05$ compared to miR-519d group. $P<0.05$ compared to AC-hMSCs-Ctrl or miR-Ctrl group.
Additional file 5 : Figure S3. The schematic diagram shows how VEGFA expression is regulated by miR-519d-3p after changes of adhesion.

\section{Abbreviations}

MSCs: Mesenchymal stem cells; MI: Myocardial infarction; hMSCs: Human MSCs; AC-hMSCs: hMSCs grown as adherent cultures; nonAC-hMSCs: hMSCs grown as nonadherent cultures on ultra-low-adherent plates; AC-VEGFAhMSCs: VEGFA-overexpressing AC-MSCs; nonAC-shVEGFA-hMSCs: VEGFAknockdown nonAC-hMSCs; RT-qPCR: Real-time quantitative polymerase chain reaction; ELISA: Enzyme-linked immunosorbent assays; HUVECs: Human umbilical vein endothelial cells; Sca-1: Stem cell antigen 1; LADCA: Left anterior descending coronary artery; LV: Left ventricle; LVEDD: LV enddiastolic dimensions; LVESD: LV end-systolic dimensions

\section{Acknowledgements}

The authors thank the volunteers who donated the bone marrow for this study.

\section{Authors' contributions}

BD collected the samples, performed the experiments, and drafted the manuscript. YL analyzed the data and prepared figure WD designed the research and revised the manuscript. $\mathrm{HJ}$ participated in the experiments. WH and YW participated in the study design. The authors read and approved the final version of manuscript.

\section{Funding}

This work was supported by the Science and Technology Planning Project of Guangdong Province (No. 2016B1030) and the Guang Dong Medical Research Foundation (No. A2016430).

\section{Availability of data and materials}

All data generated and/or analyzed during the current study are included in this manuscript. 


\section{Ethics approval and consent to participate}

All experiments were approved by the Ethics Committee of the Affiliated Hospital of Guilin Medical University, and informed consent was obtained from all participants.

\section{Consent for publication}

Not applicable

\section{Competing interests}

The authors declare that they have no competing interests.

\section{Author details}

'Department of Cardiothoracic Surgery, Zhongshan People's Hospital, 2 Sunwen East Road, Zhongshan, Guangdong 528403, People's Republic of China. ${ }^{2}$ Department of Vascular Surgery, Affiliated Hospital of Guilin Medical University, Guilin 541001, People's Republic of China. ${ }^{3}$ Department of Gastroenterology, Taihe Hospital, Hubei University of Medicine, 32 Ren Min South Road, Shiyan 442000, Hubei, People's Republic of China.

\section{Received: 9 April 2020 Revised: 12 June 2020}

Accepted: 18 June 2020 Published online: 02 July 2020

\section{References}

1. Zhao J, Li X, Hu J, Chen F, Qiao S, Sun X, et al. Mesenchymal stromal cellderived exosomes attenuate myocardial ischaemia-reperfusion injury through miR-182-regulated macrophage polarization. Cardiovasc Res. 2019; 115(7):1205-16.

2. Huang F, Zhu X, Hu XQ, Fang ZF, Tang L, Lu XL, et al. Mesenchymal stem cells modified with miR-126 release angiogenic factors and activate Notch ligand Delta-like-4, enhancing ischemic angiogenesis and cell survival. Int J Mol Med. 2013;31(2):484-92.

3. Wang N, Chen C, Yang D, Liao Q, Luo H, Wang X, et al. Mesenchymal stem cells-derived extracellular vesicles, via miR-210, improve infarcted cardiac function by promotion of angiogenesis. Biochim Biophys Acta Mol basis Dis. 2017:1863:2085-92.

4. Huang P, Wang L, Li Q, Tian X, Xu J, Xu J, et al. Atorvastatin enhances the therapeutic efficacy of mesenchymal stem cells derived exosomes in acute myocardial infarction via up-regulating long non-coding RNA H19. Cardiovasc Res. 2020:116(2):353-67.

5. Han Y, Li X, Zhang Y, Han Y, Chang F, Ding J. Mesenchymal stem cells for regenerative medicine. Cells. 2019;8(8):886.

6. Ji ST, Kim H, Yun J, Chung JS, Kwon SM. Promising therapeutic strategies for mesenchymal stem cell-based cardiovascular regeneration: from cell priming to tissue engineering. Stem Cells Int. 2017:2017:3945403.

7. Huang F, Li ML, Fang ZF, Hu XQ, Liu QM, Liu ZJ, et al. Overexpression of MicroRNA-1 improves the efficacy of mesenchymal stem cell transplantation after myocardial infarction. Cardiology. 2013;125(1):18-30.

8. Dou L, Wu Y, Yan Q, Wang J, Zhang Y, Ji P. Secretome profiles of immortalized dental follicle cells using iTRAQ-based proteomic analysis. Sci Rep. 2017:7(1):7300.

9. Rombouts WJ, Ploemacher RE. Ploemacher, primary murine MSC show highly efficient homing to the bone marrow but lose homing ability following culture. Leukemia. 2003;17(1):160-70.

10. Halfon S, Abramov N, Grinblat B, Ginis I. Ginis, markers distinguishing mesenchymal stem cells from fibroblasts are downregulated with passaging. Stem Cells Dev. 2011;20(1):53-66.

11. Barbash IM, Chouraqui P, Baron J, Feinberg MS, Etzion S, Tessone A, et al. Systemic delivery of bone marrow-derived mesenchymal stem cells to the infarcted myocardium: feasibility, cell migration, and body distribution. Circulation. 2003;108(7):863-8.

12. Karp JM, Leng Teo GS. Mesenchymal stem cell homing: the devil is in the details. Cell Stem Cell. 2009;4(3):206-16.

13. Qian H, Le Blanc K, Sigvardsson M. Primary mesenchymal stem and progenitor cells from bone marrow lack expression of CD44 protein. J Biol Chem. 2012;287(31):25795-807.

14. Deng B, Deng W, Xiao P, Zeng K, Zhang S, Zhang H, et al. Nonadherent culture method downregulates stem cell antigen-1 expression in mouse bone marrow mesenchymal stem cells. Exp Ther Med. 2015:10(1):31-6.

15. Li SH, Sun L, Yang L, Li J, Shao Z, Du GQ, et al. Young bone-marrow Sca-1+ stem cells rejuvenate the aged heart and improve function after injury through PDGFRß-Akt pathway. Sci Rep. 2017;7:41756.
16. Deng $B$, Jiang $H$, Zeng $K$, Liang $Y$, Wu Y, Yang $Y$. Removal from adherent culture contributes to apoptosis in human bone marrow mesenchymal stem cells. Mol Med Rep. 2017;15(6):3499-506.

17. Moon HH, Joo MK, Mok H, Lee M, Hwang KC, Kim SW, et al. MSC-based VEGF gene therapy in rat myocardial infarction model using facial amphipathic bile acid-conjugated polyethyleneimine. Biomaterials. 2014; 35(5):1744-54.

18. Sun L, Cui M, Wang Z, Feng X, Mao J, Chen $P$, et al. Mesenchymal stem cells modified with angiopoietin-1 improve remodeling in a rat model of acute myocardial infarction. Biochem Biophys Res Commun. 2007;357(3):779-84.

19. Lapeyre-Prost A, Terme M, Pernot S, Pointet AL, Voron T, Tartour E, et al. Immunomodulatory activity of VEGF in Cancer. Int Rev Cell Mol Biol. 2017; 330:295-342.

20. Zou J, Fei Q, Xiao H, Wang H, Liu K, Liu M, et al. VEGF-A promotes angiogenesis after acute myocardial infarction through increasing ROS production and enhancing ER stress-mediated autophagy. J Cell Physiol. 2019:234(10):17690-703.

21. Law PK, Haider K, Fang G, Jiang S, Chua F, Lim YT, et al. Human VEGF165myoblasts produce concomitant angiogenesis/myogenesis in the regenerative heart. Mol Cell Biochem. 2004;263(1-2):173-8.

22. Pons J, Huang Y, Arakawa-Hoyt J, Washko D, Takagawa J, et al. VEGF improves survival of mesenchymal stem cells in infarcted hearts. Biochem Biophys Res Commun. 2008;376(2):419-22.

23. Kim SH, Moon HH, Kim HA, Hwang KC, Lee M, Choi D. Hypoxia-inducible vascular endothelial growth factor-engineered mesenchymal stem cells prevent myocardial ischemic injury. Mol Ther. 2011;19(4):741-50.

24. Zhou K, Zhang C, Yao H, Zhang X, Zhou Y, Che Y, et al. Knockdown of long non-coding RNA NEAT1 inhibits glioma cell migration and invasion via modulation of SOX2 targeted by miR-132. Mol Cancer. 2018;17(1):105.

25. Qiu H, Wu H, Ma J, Cao H, Huang L, Qiu W, et al. DL-3-n-Butylphthalide reduces atrial fibrillation susceptibility by inhibiting atrial structural remodeling in rats with heart failure. Naunyn Schmiedeberg's Arch Pharmacol. 2018;391(3):323-34

26. Hou Y, Ryu $\mathrm{CH}$, Jun JA, Kim SM, Jeong $\mathrm{CH}$, Jeun SS. IL-8 enhances the angiogenic potential of human bone marrow mesenchymal stem cells by increasing vascular endothelial growth factor. Cell Biol Int. 2014; 38(9):1050-9.

27. Song YS, Joo HW, Park $I H$, Shen GY, Lee $Y$, Shin JH, et al. Bone marrow mesenchymal stem cell-derived vascular endothelial growth factor attenuates cardiac apoptosis via regulation of cardiac miRNA-23a and miRNA-92a in a rat model of myocardial infarction. PLoS One. 2017;12(6): e0179972.

28. Tang Y, Gan X, Cheheltani R, Curran E, Lamberti G, Krynska B, et al. Targeted delivery of vascular endothelial growth factor improves stem cell therapy in a rat myocardial infarction model. Nanomedicine. 2014;10(8):1711-8.

29. Kang K, Ma R, Cai W, Huang W, Paul C, Liang J, et al. Exosomes secreted from CXCR4 overexpressing mesenchymal stem cells promote cardioprotection via Akt signaling pathway following myocardial infarction. Stem Cells Int. 2015:2015:659890.

30. Ferrara N, Gerber HP, LeCouter J. The biology of VEGF and its receptors. Nat Med. 2003;9(6):669-76.

31. Markel TA, Wang Y, Herrmann JL, Crisostomo PR, Wang M, Novotny NM, et al. VEGF is critical for stem cell-mediated cardioprotection and a crucial paracrine factor for defining the age threshold in adult and neonatal stem cell function. Am J Physiol Heart Circ Physiol. 2008;295(6):H2308-14.

32. Cho HM, Kim PH, Chang HK, Shen YM, Bonsra K, Kang BJ, et al. Targeted genome engineering to control VEGF expression in human umbilical cord blood-derived Mesenchymal stem cells: potential implications for the treatment of myocardial infarction. Stem Cells Transl Med. 2017:6(3):1040-51.

33. Dai Y, Xu M, Wang Y, Pasha Z, Li T, Ashraf M. HIF-1alpha induced-VEGF overexpression in bone marrow stem cells protects cardiomyocytes against ischemia. J Mol Cell Cardiol. 2007:42(6):1036-44.

34. Linde N, Lederle W, Depner S, van Rooijen N, Gutschalk CM, Mueller MM. Vascular endothelial growth factor-induced skin carcinogenesis depends on recruitment and alternative activation of macrophages. J Pathol. 2012;227(1): 17-28.

35. Engel JE, Williams E, Williams ML, Bidwell GL, Chade AR. Targeted VEGF (vascular endothelial growth factor) therapy induces long-term renal recovery in chronic kidney disease via macrophage polarization. Hypertension. 2019;74(5):1113-23. 
36. Li Y, Zheng D, Pan L, Dai Y, Cai S, Zhao L, et al. Knockdown of TUG1 by shRNA inhibited renal cell carcinoma formation by miR-299-3p/NEGF axis in vitro and in vivo. Eur J Pharmacol. 2019;860:172536.

37. Hou J, Wang L, Wu Q, Zheng $\mathrm{G}$, Long $\mathrm{H}$, Wu H, et al. Long noncoding RNA H19 upregulates vascular endothelial growth factor A to enhance mesenchymal stem cells survival and angiogenic capacity by inhibiting miR199a-5p. Stem Cell Res Ther. 2018;9(1):109.

38. Bentwich I, Avniel A, Karov Y, Aharonov R, Gilad S, Barad O, et al. Identification of hundreds of conserved and nonconserved human microRNAs. Nat Genet. 2005;37(7):766-70.

39. Hua KT, Hong JB, Sheen YS, Huang HY, Huang YL, Chen JS, et al. miR-519d promotes melanoma progression by downregulating EphA4. Cancer Res. 2018;78(1):216-29

40. Fornari F, Milazzo M, Chieco P, Negrini M, Marasco E, Capranico G, et al. In hepatocellular carcinoma miR-519d is up-regulated by p53 and DNA hypomethylation and targets CDKN1A/p21, PTEN, AKT3 and TIMP2. J Pathol. 2012;227(3):275-85.

41. Ye X, Lv H. MicroRNA-519d-3p inhibits cell proliferation and migration by targeting TROAP in colorectal cancer. Biomed Pharmacother. 2018;105:87986.

42. Zhang SJ, Chen X, Li CP, Li XM, Liu C, Liu BH, et al. Identification and characterization of circular RNAs as a new class of putative biomarkers in diabetes retinopathy. Invest Ophthalmol Vis Sci. 2017;58(14):6500-9.

\section{Publisher's Note}

Springer Nature remains neutral with regard to jurisdictional claims in published maps and institutional affiliations.

Ready to submit your research? Choose BMC and benefit from:

- fast, convenient online submission

- thorough peer review by experienced researchers in your field

- rapid publication on acceptance

- support for research data, including large and complex data types

- gold Open Access which fosters wider collaboration and increased citations

- maximum visibility for your research: over $100 \mathrm{M}$ website views per year

At BMC, research is always in progress.

Learn more biomedcentral.com/submissions 